\title{
Implementing Remote Presence Using Quadcopter Control by a Non-Invasive BCI Device
}

\author{
Jzau-Sheng Lin ${ }^{*}$, Zi-Yang Jiang \\ Department of Computer Science and Information Engineering, National Chin-Yi University of Technology, Taiwan
}

Copyright $@ 2015$ Horizon Research Publishing All rights reserved.

\begin{abstract}
Extracting neural signals to control a quadcopter using wireless manner is proposed in this paper for hands-free, silence and effortless human-mobile interaction with remote presence. The brain activity is recorded in real-time and discovered patterns to relate it to facial-expression states with a cheap off-the-shelf electroencephalogram (EEG) headset-Emotic Epoc device. A tablet based mobile framework with Android system is developed to convert these discovered patterns into commands to drive the quadcopter-AR Drone 2.0 through wireless interface. First, neural signals are sequentially extracted from headset and transmitted to the tablet mobile system. In the tablet mobile system, large number of feature vector of EEG can be reduced by using Principle Component Analysis (PCA) to recognize the facial expression to generate suitable commands and driving the quadcopter through wireless interface. Finally, the quadcopter can fly smoothly in accordance with the commands converted by the EEG signals. The experimental results show that the proposed system can easily control quadcopters.
\end{abstract}

\section{Keywords EEG, BCI, PCA}

\section{Introduction}

Instead of clinic-used BCI headset system, the recent availability of portable low-cost wireless EEG headsets is focused in the applications of several fields such as electric wheelchair, computer games, mobile phones, robot arms, and so on. In accordance with the extracted neural signals, embedding low-cost EEG sensors into wireless headsets gives improved authentication and becomes a practical opportunity. There are several techniques like electroencephalogram (EEG), Magnetoencephalogram (MEG), functional Magnetic Resonance Imaging (fMRI), Positron Emission Tomography (PET), Diffusion Tensor Imaging (DTI), and Near-Infra-Red Spectroscopy (NIRS) have been utilized to extracted neural signals for analyzing the thinking, behaviors, and diseases of human. Each technology has its own advantages and disadvantages. EEG measurement is inexpensive, non-invasive and popular Brain-Computer Interface (BCI) instrumentation with highly accurate recording and exceptional time resolution for neural signals extraction.

In the research of signal transformation in brain science, BCI system is created to obtain the human EEG signals in order to build an interactive system, and converted them into commands that enable advanced algorithms, or computer system to identify and deal with these commands. $\mathrm{BCI}$ provides a communication channel that allows the user by the strength of brain wave signals to communicate with the outside world through the brain activity to directly infer the subject's intention to transform into a computer-controlled signal. Communication and control are some of them, notably by allowing to spell words, to use a web browser or to control a system just by thought. Several research areas are the study of motor substitution or motor rehabilitation whose main applications are hand grasping [1], Short Message Service (SMS) in mobile phone [2], and wheelchair control [3, 4]. Additionally, for healthy end-users, BCIs have also been used to augment interactivity in games by using multimodality from the EEG signals $[5,6]$.

A well-established tool in studying the neural-function correlates of cognitive processes, the EEG provides much-needed insight into human brain. An EEG headset has several advantages like wireless manner with Bluetooth or $\mathrm{Wi}-\mathrm{Fi}$, fast data refresh rate, real-time data acquisition and exchange in a well-organized system. Additionally, it plays a role in diagnosis and health management with low cost. While bearing in mind for short-time development of EEG-based BCI, low-cost devices for brain wave extraction with uncomplicated mechanisms and less time utilization is required. NeuroSky Mindset [7] has fixed position of Fp1 and one reference channel A1. It can extract brain waves only. Emotive Epoc neuro, a low-cost headset, has 14 electrodes with 2 reference channels, in which revealing of blinks, horizontal glances left/right, eyebrow raise, left/right winks, smile left/right, laugh, clench teeth and several expressions as well as cognitive states can be real-time recorded. 
There have been some applications that successfully utilize this technology in several fields [8-11]. In 2013, Duvinage et al. [8] proposal a BCI system to discussed the performance of the Emotiv Epoc headset for P300-based applications. Ramirez and Vamvakousis [9] used Emotive Epoc device to detect emotion from EEG signals. They extracted features from the EEG signals in order to characterize states of mind in the arousal-valence 2D emotion model. Using these features they classified EEG signals into high/low arousal and positive/negative valence emotional states. Szar and Signorile [10] used Emotiv system to extract the EEG signals from the headset to categorize them into one of several groups, to translate that group to a robotic command, and finally controlled the robot. Thobbi et al. [11] achieved remote presence using the Emotiv Epoc headset to control a humanoid robot.

Because of the application of Unmanned Aerial Vehicle (UAV) and Micro Aerial Vehicle (MAV) can apply to variety of area such as military, rescue mission, film making, agriculture and others, research and development of UAV and MAV are recently getting high encouragement. Quadcopter is one of the UAV that is major focuses of active researches in recent years. In 2012, a low-cost hover control mechanism was developed and implemented on a low-cost microcontroller for a quadcopter [12]. Hanafi et al. [13] presented the development of remotely controlled quadcopter system through a Graphical User Interface (GUI) where the communication between GUI and quadcopter is constructed by using wireless communication system. In this paper, remote presence was implemented to control a quadcopter (AR Drone 2.0) [14] by the Emotiv EPOC [15] device. The motivation in this paper is that to develop a non-invasive BCI device to control suitable systems, such as wheelchair or electrical bed, through the experimental results in the quadcopter control.

PCA [16], an orthogonal-transformation procedure, is used to convert a set of observations of possibly correlated data into a set of values of linearly uncorrelated data called principal components. The number of principal components is less than or equal to the number of original data. The PCA was used to reduce the data dimension of EEG signal in this paper.

This paper is organized as follows. The system architecture is introduced in Section 2. Section 3 shows the implementation of the proposed system. Section 4 demonstrates the feature extraction, Experimental results are displayed in Section 5, Finally, Section 6 is the discussion and conclusions.

\section{System Architecture}

The proposed remote presence to control the AR Drone 2.0 quadcopter by the Emotive EPOC device is shown as in Fig. 1. The architecture includes an EEG signal acquisition unit with 16-elctrode headset, signal processing unit with the development kit EmoEngine, Emotiv API, wireless interface, and a wireless-controlled quardcopter.

\section{Implementation}

\subsection{Emotiv EPOC device}

In the proposed system, the Emotiv EPOC headset, shown as in Fig. 2, is used to extract the EEG signals. It consists of 14-channel bio potential sensors with gold-plated electrodes offer optimal positioning for accurate spatial resolution. Additionally, CMS/DRL reference locations are also utilized. Based on the international 10-20 locations, these 14 EEG names are AF3, AF4, F7, F8, F3, F4, FC5, FC6, T7, T8, P7, P8, O1, and O2 which are shown as in Fig. 2. In the Emotiv headset, the sampling rate is 128 $\mathrm{Hz}$ on the output as well as the internal sampling rate is $2048 \mathrm{~Hz}$ with 1.95- $\mu \mathrm{V}$ Least Significant Bit (LSB) voltage resolution. The extracted 14-channel waveforms are shown as in Fig. 3.

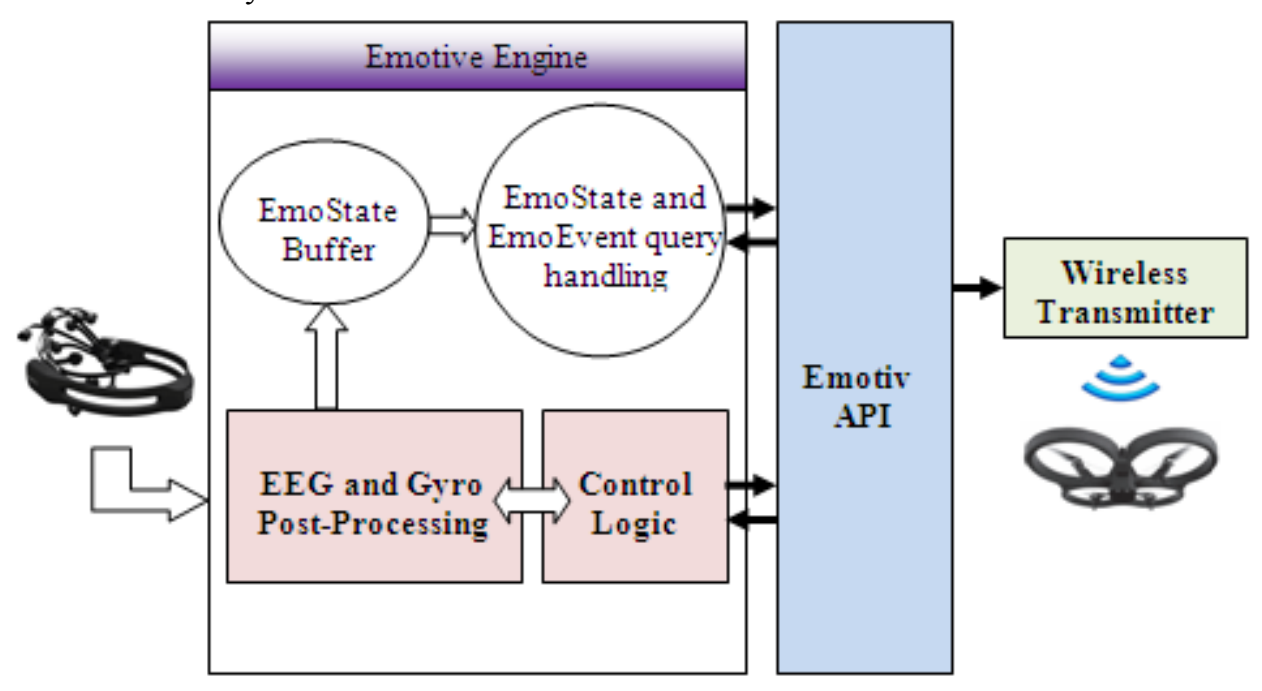

Figure 1. The proposed system diagram 


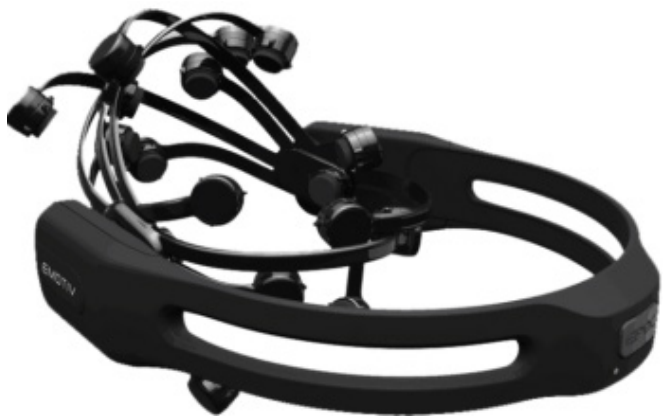

(a)

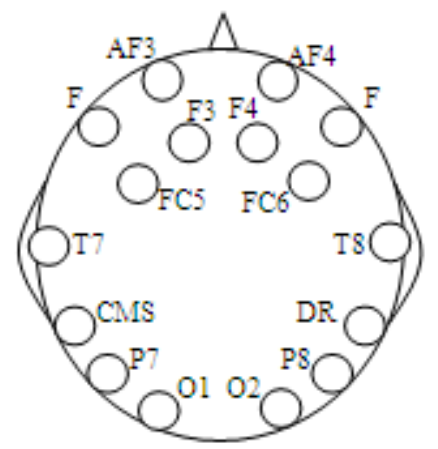

(b)

Figure 2. (a) Emotiv Epoc; (b) Locations of Emotiv electrodes
In the Emotiv headset, EPOC control panel collects sequentially sampled data and supplies the data to an application. Three built-in outputs, named Affective, Cognitive, and Expressive suits are processed. Affective suit detects positive mental states such as concentration, meditation and excitement. Cognitive suit stores user's neutral or relaxed mental states. Expressive suit measures facial movements and detects different states such as left/right smile, raise/frown brow, left/right wink, etc.

\subsection{Quardcopter Subsystem}

The quadcopter model used in this research is the AR Drone 2.0 Quadflyer. The quadcopter subsystem consists of a receiver module with wireless interface, a gyroscope unit, Ultrasonic Height Sensor (UHS), accelerometer circuits, four propeller DC motors and a Lithium-Polymer battery. Fig. 4 shows the components of the quadcopter subsystem. The command signal is a Pulse-Width Modulated (PWM) signal that is received by the receiver module. The gyroscope unit will modify received signals by incorporating the attitude (roll, pitch and yaw angles) information before transmitting the signal to the accelerometer circuits. The UHS provides a value of the distance between the bottom of the drone and the object below. The accelerometer circuits give the drone an awareness of its acceleration in each direction of the four DC motors.
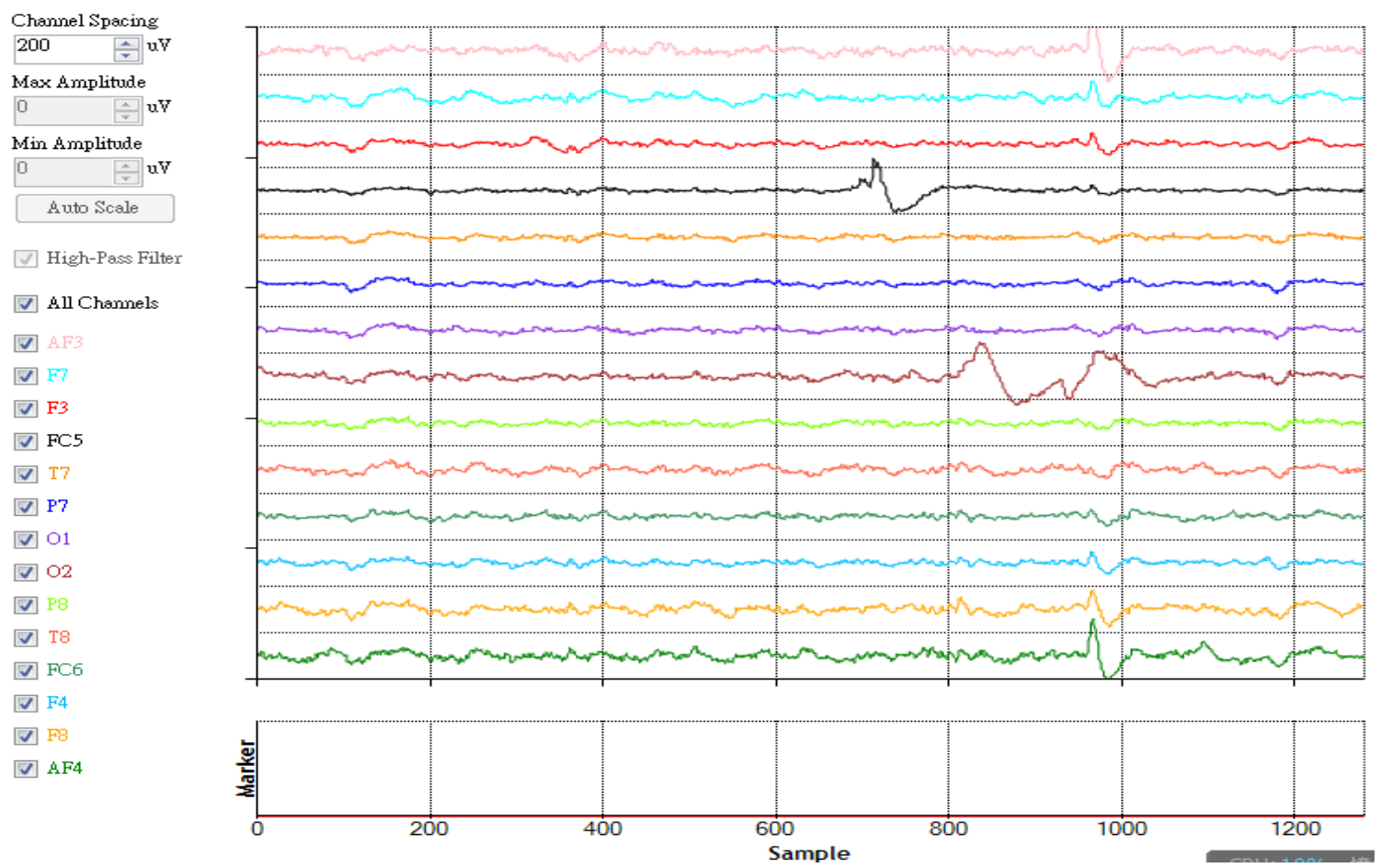

Figure 3. Extracted brain waves from 14 channels on Emotive headset 


\section{Feature Extraction}

In the proposed system, we extracted facial states such as left/right smile, raise/frown brow, and left/right wink from 6 locations such as AF3, AF4, F3, F4, F7, and F8.

PCA is an approach used to condense the properties of multivariate analysis in a set of data samples. It is a linear transformation scheme frequently used for feature extraction or data compression. PCA, also known as the Karhunen-Loeve transformation [17] in communication theory, maximizes the rate of decrease of variance. From the viewpoint of statistical pattern recognition, the practical value of PCA is that it supplies an effective way for dimensionality reduction. The number of features needed for effective data representation can be reduced. On the other hand, it discards those terms that have small variance and retains only those terms that have large variance [18].

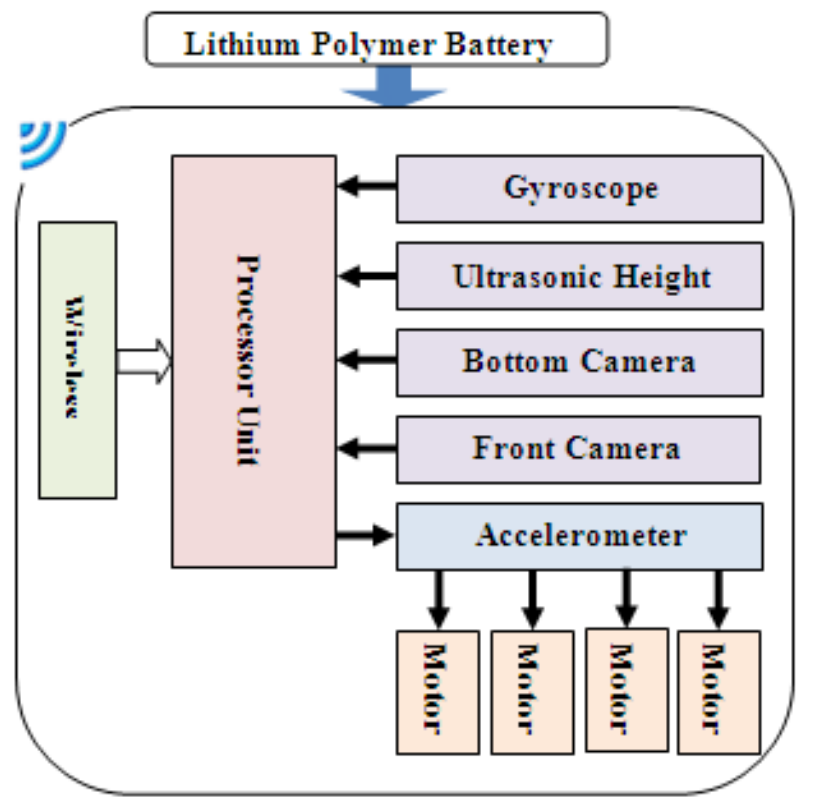

Figure 4. System architecture of AR Drone 2.0 quadflyer

PCA technique can be modeled as follows. There exists $k$ samples with $n$-dimensional input vectors $\mathbf{X}_{1}, \mathbf{X}_{2}, \ldots, \mathbf{X}_{k} \in \mathbf{R}^{n}$. The expected vector is defined as

$$
\boldsymbol{\psi}=\sum_{i=1}^{k} \mathbf{X}_{i}
$$

An array $\left[\boldsymbol{\Phi}_{1}, \Phi_{2}, \ldots . ., \Phi_{k}\right]$ is calculated where $\boldsymbol{\Phi}_{i}$ is defined as the difference between $\mathbf{X}_{i}$ and $\boldsymbol{\Psi}$. Then the covariance matrix can be computed as follows

$$
\mathbf{C}=\boldsymbol{\Phi} \boldsymbol{\Phi}^{T}
$$

Then the first $m(m<n)$ eigenvectors of $\mathbf{C}$ are named as principle components and computed. For different facial expression states, different principle components can be extracted. When an unknown EEG is extracted, we can compute its covariance matrix and principle components. Finally, the unknown principle components are compared with those principle components of different facial expression states to indicate which state is displayed.

\section{Experimental Results}

In this study, eight healthy subjects with an average age of 23.3 years old were participated in experiments. For these eight subjects, we extracted EEG signals form AF3, $\mathrm{AF} 4, \mathrm{~F} 3, \mathrm{~F} 4, \mathrm{~F} 7$, and F8 locations to collect six sets indicated six facial expression states such as left/right smile, raise/frown brow, and left/right wink. And every set occupies 20 principle components. The definition of control commands for the flying quadcopter is shown as in Table I.

The Information Transmission Rate (ITR) is generally used to estimate the performance of the communication and control for brain-computer interfaces [19]. The higher value indicates the more performance. The proposed method is also used ITR to assess the performance of the system. The ITR is defined by

$$
\begin{gathered}
\frac{\text { Bits }}{\text { Command }}=\log _{2} N+P \log _{2} P+(1-P) \log _{2} \frac{1-P}{N-1} \\
\text { ITR }=\frac{\text { Bits }}{\text { Command }} \cdot d_{r}
\end{gathered}
$$

where $N$ is the total number of commands ( $N=6$ in our system), $P$ is the accuracy, and $d_{r}$, expressed as decision rate, is the average commands during an unit time.

Table 1. Control commands for the flying quadcopter

\begin{tabular}{|c|c|c|}
\hline $\begin{array}{c}\text { Control } \\
\text { commands }\end{array}$ & left right \\
\hline smile & fly left & \\
\hline brow & fB fly right \\
\hline wink & forward & \\
\hline
\end{tabular}

In the experimental scenario, we defined 4 points from take-off to loading locations in a 3-D open area. Every subject had to finished complete path from start location to end location through these 4 points with 3 times. The average ITRs for different subjects can obtain suitable values. 


\section{Discussion and Conclusions}

In this paper, a Non-Invasive BCI device with expressive manner on face is implemented in remote presence using quadcopter control with Emotive EPOC headset. For subjects, EEG signals were extracted from locations on AF3, AF4, F3, F4, F7, and F8 respectively. The principle components were detached from these six EEGs to transfer one of six commands such as left/right smile, raise/frown brow, and left/right wink to control a quadcopter. From the experimental results, the proposed strategy can be suitably used to naturally control a quadcopter through this low-cost wearable device.

\section{Acknowledgements}

In this paper, the research was sponsored by the National Science Council of Taiwan under the Grant NSC103-2221-E-167 -027-.

\section{REFERENCES}

[1] Pfurtscheller G., Müller G. R., Pfurtscheller J., Gerner H. J., and Rupp R. 2003. Thought' - Control of Functional Electrical Stimulation to Restore Hand grasp in a Patient with Tetraplegia, Neurosci Lett, 351, 33-36.

[2] Lin J.-S., Wang M., Lia P.-Y., and Li Z. 2014. An SSVEP-Based BCI System for SMS in a Mobile Phone, Applied Mechanics and Materials, 513-517, 412-415, 2014.

[3] Lin J.-S., Wang M., and Hsieh C.-H. 2014. An SSVEP-Based BCI System with SOPC Platform for Electric Wheelchairs, Trans. On Computer Science and Technology, $3(2), 35-40$.

[4] Lin J.-S., and Huang S.-M. 2013. An FPGA-Based Brain-Computer Interface for Wireless Electric Wheelchairs, Applied Mechanics and Materials, 283-287, 1616-1621.

[5] Nijholt A., Bos D. P. O., and Reuderink B. 2009. Turning Shortcomings into Challenges: Brain-Computer Interfaces for Games," Entertainment Comput., 1(2), 85-94.

[6] Mühl C., GürkökH, H. Plass-Oude Bos D., Thurlings M., Scherffig L., Duvinage M., Elbakyan A., Kang S., Poel M., and Heylen D. 2010. "Bacteria Hunt," J. of Multimodal. User Interfaces, 4, 11-25.
[7] Hal B. Van, Rhodes S., Dunne B., Bossemeyer R., Low-Cost EEG-based Sleep Detection, The 36th IEEE Annual Int. Conf., 4571-4574, 2014.

[8] Duvinage M., Castermans T., Petieau M., Hoellinger T., Cheron G., and Dutoit T. 2013. Performance of the Emotiv Epoc Headset for P300-Based Applications, BioMedical Engineering OnLine, 12:56, http://www.biomedical-enginee ring-online.com /content/12/1/56.

[9] Ramirez R. and Vamvakousis Z. 2012. Detecting Emotion from EEG Signals Using the Emotive Epoc Device, Lecture Notes in Computer Science, 7670, 175-184.

[10] Szar D. and Signorile R., An Exploration of the Utilization of Electroencephalography and Neural Nets to Control Robots, Human-Computer Interaction- INTERACT 2011, 186-194, 2011.

[11] Thobbi A., Kadam R., and Sheng W. 2010. Achieving Remote Presence Using a Humanoid Robot Controlled by a Non-Invasive BCI Device, Int. J. on Artificial Intelligence and Machine Learning, 10, 41-45.

[12] Leong B. T. M., Low S. M., and Ooi M. P.-L. 2012. Low-Cost Microcontroller-based Hover Control Design of a Quadcopter, Procedia Engineering, 41, 458 - 464.

[13] Hanafi D., Qetkeaw M., Ghazali R., Than M. N. M., Utomo W. M., and Omar R. 2013. Simple GUI Wireless Controller of Quadcopter, Int. J. Communications, Network and System Sciences, 6, 52-59.

[14] “ARDrone_Developer_Guide", https://projects.ardrone.org/wiki/ardrone-api/Developer_Gu ide.

[15] Stytsenko K, Jablonskis E, Prahm C, Evaluation of consumer EEG Device Emotiv EPOC, Proceedings of the MEi:CogSci Conference; 2011.

[16] Lin J.-S. and Liu S.-H., 2004. Still Color Images Encoding through a Rough PCA Net and Interpolative Approaches with a Feed Forward Net, Int. J. of electrical Engineering, 11, 87-95.

[17] Effros M. and Chou P., Weighted Universal Transform Coding: Universal Image Compression with the Karhunen-Loeve Transform, Proc. Of Int. Conf. on Image Processing, 61-64, 1995.

[18] Oja E., Subspace Methods of Pattern Recognition, Letchworth, UK:Research Studies Press, 1983.

[19] Wolpaw J. R., Birbaumer N., McFarland D. J., Pfurtscheller G., and Vaughan, T. M. 2002. Brain-Computer Interfaces for Communication and Control, Clinical Neurophysiology, 113, 67-79. 